\title{
Very light Physical Activity after a Meal Blunts the Rise in Blood Glucose and Insulin
}

\author{
Eivind Aadland ${ }^{* 1}$ and Arne T. Høstmark ${ }^{1,2}$ \\ ${ }^{I}$ The Norwegian School of Sport Sciences, Postbox 4014 Ullevål Stadion, 0806 Oslo, Norway \\ ${ }^{2}$ Section of Preventive Medicine and Epidemiology, Institute of General Practice and Community Medicine, University \\ of Oslo, Norway
}

\begin{abstract}
The objective of this study was to examine the impact of post-meal light exercise on the rise in blood glucose and insulin. After fasting overnight, nine healthy subjects (age $37.3 \pm 12.2$ years) participated in three experiments in a crossover design: Day 1 (no exercise), the subjects were given cornflakes, and blood glucose and plasma insulin were determined before meal, and each 15 min for the next $165 \mathrm{~min}$. Day 2 and 3 were similar to Day 1, but included 30 min very light and light intensity bicycle exercise after the meal. Both levels of exercise blunted and delayed the rise in blood glucose and plasma insulin. Bicycling-related reductions in peak glucose and insulin values correlated with peak values the control day ( $\mathrm{r}=-0.83$ to $-0.93, \mathrm{P} \leq 0.006$ ), as did reductions in AUC and IAUC. We conclude that even very light exercise after a carbohydrate meal blunts the rise in blood glucose and insulin.
\end{abstract}

Keywords: Glucose, insulin, exercise, postprandial.

\section{INTRODUCTION}

The prevalence of impaired glucose tolerance and type 2 diabetes is increasing [1] and it is estimated that the number of individuals with the disease will double from year 2000 to 2030 [2]. The cost of treatment is high and type 2 diabetes is characterized as one of the main health concerns in the 21.century [3]. Both high fasting glycaemia and high postprandial glycaemia increase the risk of morbidity and mortality $[4,5]$, and some studies suggest that postprandial glycaemia may be the most important of the two [6-8]. Longitudinal studies have shown that intake of acarbose, an alpha glucosidase inhibitor, decreases the incidence of type 2 diabetes and cardiovascular disease [9-11]. Hanefeld et al. [12] found a total reduction in incidence of cardiovascular disease of $35 \%$ in a metaanalysis of seven randomized controlled trials using acarbose three times a day over a mean period of 403 days. It is now generally agreed on that high postprandial glycaemia should be reduced in susceptible individuals [13-18].

The research as to what extent exercise performed immediately after a meal can reduce postprandial glycaemia is scarce. It has been reported that light exercise blunts the increase in both glycaemia and insulinaemia after a meal [19, 20]. To our knowledge, the study of Høstmark et al. [20] is the only one to examine the effect of exercise performed immediately after a carbohydrate meal on the postprandial blood glucose concentration. The study examined the effect of 30 min light bicycle exercise $(\sim 70 \%$ of maximal heart rate $\left.\left[\mathrm{HR}_{\max }\right]\right)$ initiated immediately after consumption of a high glycaemic meal (cornflakes) in 19 young and 20 middle-aged women. There was a consistent decrease in blood

*Address correspondence to this author at the Loftesnes Hytter, 6856 Sogndal, Norway; E-mail: eaadland@ hotmail.com glucose in the time interval when the exercise was executed, and a greater benefit provided by the work for middle-aged, compared to young women. Since glucose tolerance is negatively associated with age [21], this could mean that the effect of work in the postprandial period depends on the glucose tolerance of each and every individual, as shown in rats [22]. Hence, one of the objectives in the present study was to examine this relationship in humans. Furthermore, the effect of the intensity of the work performed is unsettled [23]. Achten \& Jeukendrup [23] found no difference in blood glucose or insulin between work of differing intensities $(\sim 55,77$ and $90 \%$ of maximal oxygen consumption) performed by eight men 45 min after intake of $75 \mathrm{~g}$ carbohydrate. Consequently, the other objective of our study was to examine the effect of exercise at a somewhat lower intensity than used by Høstmark et al. [20], on postprandial glycaemia and insulinaemia.

\section{MATERIALS AND METHODOLOGY}

After approval by the regional ethics committee in Norway South, the participants received written information about the study and gave their written informed consent to participate. Inclusion criterion was age over 20 years and exclusion criteria were known diabetes or cardiovascular disease. Nine subjects took part in the study, six men and three women ([mean \pm SD] $37.3 \pm 12.2$ years, $77.8 \pm 14.9$ $\mathrm{kg}, 174.9 \pm 7.5 \mathrm{~cm}, 5.0 \pm 2.6$ hours of physical activity per week). The study had a crossover design. Each subject, commencing after an overnight fast, participated in three diet experiments carried out on three separate days with at least one day between each experiment. Day I served as control in which the subjects ate a high glycaemic meal (cornflakes (glycemic index (GI): $81 \pm 3$ with glucose as reference food [24] and $300 \mathrm{ml}$ skimmed milk (GI: $32 \pm 5$ )) [24], providing $1 \mathrm{~g}$ carbohydrate per $\mathrm{kg}$ body weight) in $15 \mathrm{~min}$, and then rested sitting calm for the rest of the experiment, while the 
same subjects on Day II and III carried out 30 min very light (VLI) and light (LI) bicycle-ergometer exercise on a stationary bicycle (Ergomedic 828E/ Ergomedic 818E, Monark, Sweden) immediately upon finishing the meal. The experiments were executed in this specific order, as this gave the opportunity to set the load on Day II and III after finishing the experiment on Day I. The intensities were aimed at a Borg scale of perceived exertion value of nine and 11 on Day II and III, respectively, i.e. very light (VLI) and light (LI) exercise. Body weight and height were measured every day at attendance. Venous blood samples $(\sim 5 \mathrm{ml})$ were drawn through a catheter and heart rate was measured (S610, Polar Electro Oy, Kempele, Finland) for every $15 \mathrm{~min}$ from before the meal throughout the experiment $(165 \mathrm{~min})$. In addition we noted perceived exertion on Borg scale during the work period, and self-reported weekly physical activity the last three months. Glucose was analyzed in whole blood immediately, using glucometer (Ascensia Contour, Bayer HealthCare LCC, Mishawaka, USA) and stix (Ascensia Microfill, Bayer HealthCare LCC, Mishawaka, USA). Plasma was stored at $-80^{\circ} \mathrm{C}$ until insulin analyses were carried out using an ELISA-kit (Dako Cytomation, Cambridgeshire, United Kingdom).

\section{Statistical Analyses}

Maximal heart rate was estimated by subtracting age from 220. Area under the curve (AUC) and incremental area under the curve (IAUC) were calculated using the trapezoidal rule. A two-factor repeated measures ANOVA was used to identify significant interactions between time and experiments. Repeated measures ANOVA was also used to assess differences in blood glucose (insulin) at single points of time, as well as AUC, IAUC and peak glucose (insulin) values between the three experiments. Significant differences

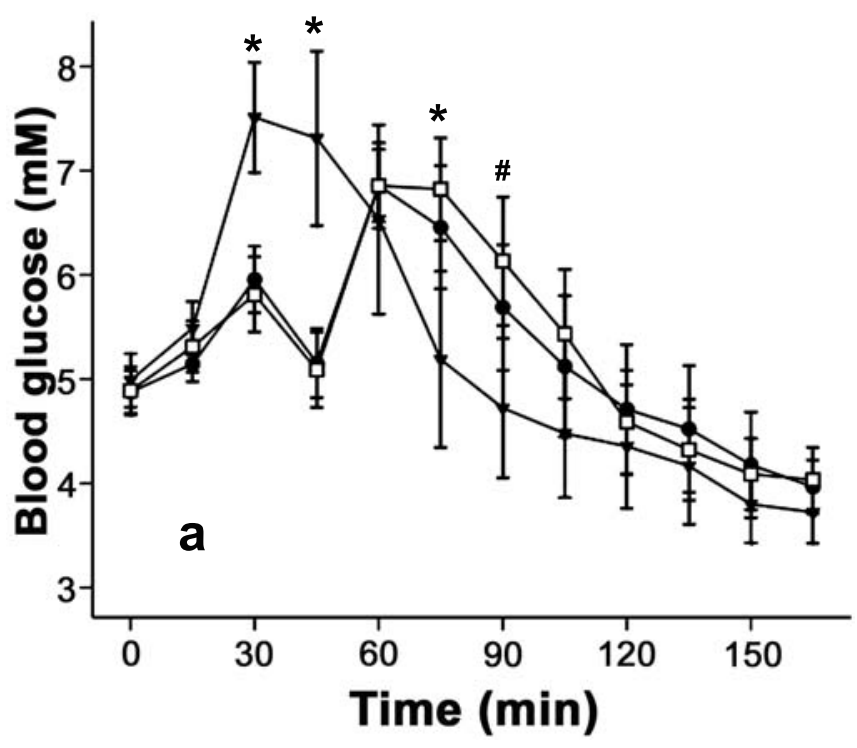

were also analyzed by pairs, using t-test for dependent groups, with Bonferroni correction. Relationships were analyzed using Pearson correlation coefficient. All analyses were performed using SPSS 15.0 (Chicago, USA). Data are presented as mean values $\pm \mathrm{SD}$. Level of statistical significance was set to $\mathrm{p} \leq 0.05$.

\section{RESULTS}

Resting heart rate was $64.8 \pm 7.5$ beats $/ \mathrm{min}$ in the control experiment, and rose to $107.1 \pm 13.3\left(58.7 \pm 6.2 \%\right.$ of $\left.\mathrm{HR}_{\max }\right)$ and $121.3 \pm 19.1$ beats/min $\left(66.6 \pm 10.6 \%\right.$ of $\left.H R_{\max }\right)$ after 30 min exercise in the VLI and LI experiment, respectively. Corresponding rates of perceived exertion were $10.1 \pm 0.9$ and $11.8 \pm 0.7$.

Fig. (1a) shows the blood glucose concentration in the three experiments. On Day I the mean peak value was reached after approximately $30 \mathrm{~min}$, and then decreased for the rest of the observation period. The postprandial increase in the glucose concentration was appreciably decreased on both days of post-meal exercise compared to control, as observed both at $30 \mathrm{~min}$ (control: $7.5 \pm 1.6$, VLI: $6.0 \pm 1.0$ [p = $0.002 v s$ control], LI: $5.8 \pm 1.1 \mathrm{mM}$ [p $=0.001 v s$ control]) and 45 min (control: $7.3 \pm 2.5$, VLI: $5.2 \pm 1.0[\mathrm{p}=0.011 \mathrm{vs}$ control], LI: $5.1 \pm 1.1 \mathrm{mM}$ [p $=0.013 v s$ control]). It seemed, however, that post meal exercise caused a displacement to the right of the blood glucose response curve, as shown by a significantly higher value at $75 \mathrm{~min}$ in both VLI $(6.5 \pm 1.8$ $\mathrm{mM}[\mathrm{p}=0.015])$ and LI experiment $(6.8 \pm 1.5 \mathrm{mM}[\mathrm{p}=$ $0.009])$ as compared to control $(5.2 \pm 2.5 \mathrm{mM})$. In the LI experiment blood glucose concentration was also significantly higher at 90 min compared to control $(6.1 \pm 1.9$ vs 4.7 $\pm 2.0 \mathrm{mM}, \mathrm{p}=0.001)$. This was also confirmed by a significant repeated measures ANOVA for interaction between

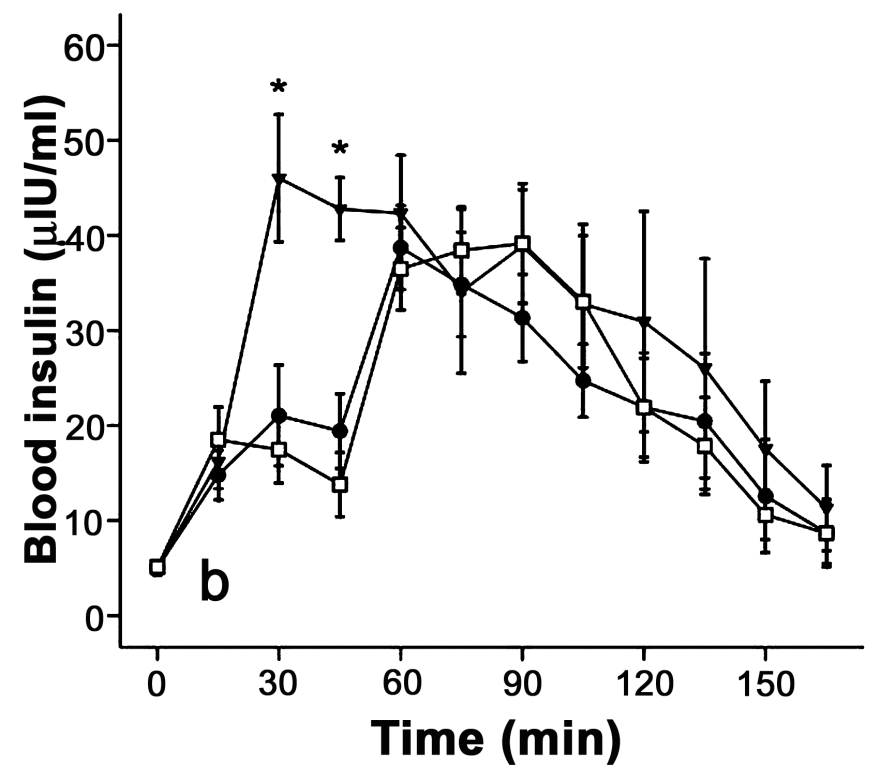

Fig. (1). Blood glucose (a) and insulin (b) concentrations throughout the three experiments carried out in the study. Nine subjects ([mean \pm SD] 37,3 $\pm 12,2$ years, 77,8 $\pm 14,9 \mathrm{~kg}, 174,9 \pm 7,5 \mathrm{~cm}, 5,0 \pm 2,6$ hours of physical activity per week) participated in three diet experiments. Commencing after an overnight fast the same subjects ate cornflakes ( $1 \mathrm{~g}$ carbohydrate per kg body weight) and then rested (control; $\boldsymbol{\nabla})$, or $\operatorname{did} 30$ min very light exercise $\left(58,7 \pm 6,2 \%\right.$ of $\left.\mathrm{HR}_{\max }\right)(\mathrm{VLI} ; \bullet)$, or $30 \mathrm{~min}$ light exercise $\left(66,6 \pm 10,6 \%\right.$ of $\left.\mathrm{HR}_{\max }\right)(\mathrm{LI}$; $\square)$ in the time period 15 to 45 min. * $\mathrm{p}<0.05$ for the difference between the control experiment and both exercise experiments; \# $<<0.05$ for the difference between the control and LI experiment. Data are shown as means \pm SEM. The study was carried out at The Norwegian School of Sport Sciences in Oslo, spring 2006. 
time and experiment $(\mathrm{F}=6.66, \mathrm{p}=0.002)$. We found no differences between the three experiments in AUC or IAUC, but a significant difference between the VLI experiment and the control experiment existed for the mean of the individual peak values $(7.2 \pm 1.5$ vs $8.1 \pm 1.1 \mathrm{mM}$, respectively $[\mathrm{p}=$ $0.006]$ ), which was reduced by $10.3 \pm 6.3 \%$. The reduction in mean of individual peak values caused by the exercise in the LI experiment was somewhat lower $(7.8 \pm 8.9 \%)$ and did not reach statistical significance after Bonferroni correction $(\mathrm{p}=0.040)$.

Insulin concentration for the three experiments is shown in Fig. (1b). After cornflake intake Day I the insulin concentration rose to reach the peak value after $30 \mathrm{~min}$ and then decreased gradually during the rest of the observation period. The insulin concentration was significantly decreased on both days of exercise both at $30 \mathrm{~min}$ (control: $46.0 \pm 18.9$, VLI: $21.0 \pm 15.0$ [p $<0.001$ vs control], LI: $17.5 \pm 9.9$ $\mu \mathrm{IU} / \mathrm{ml}[\mathrm{p}=0.001 \mathrm{vs}$ control]) and at $45 \mathrm{~min}$ (control: $42.8 \pm$ 9.8, VLI: $19.4 \pm 11.8$ [p $=0.001$ vs control], LI: $13.8 \pm 10.0$ $\mu \mathrm{IU} / \mathrm{ml}$ [p $<0.001 v s$ control]), as compared to control. A repeated measures ANOVA showed a significant interaction of time and experiment $(\mathrm{F}=3.51, \mathrm{p}=0.047)$, which means that the work caused a shift in the insulin curves to the right. In addition, we found significant differences between the VLI and control experiment in AUC (3655.8 $\pm 1834.6 v s$ $4905.1 \pm 2807.1(\mu \mathrm{IU} / \mathrm{ml}) * \min$, respectively $[\mathrm{p}=0.014])$, IAUC $(2751.2 \pm 1588.1$ vs $4067.3 \pm 2539.7(\mu \mathrm{IU} / \mathrm{ml}) * \min$, respectively $[\mathrm{p}=0.010])$ and peak value $(44.3 \pm 15.1 \mathrm{vs} 68.3$ $\pm 27.4 \mu \mathrm{IU} / \mathrm{ml}$, respectively $[\mathrm{p}=0.005])$, in which the decreases were $24.8 \pm 12.2,31.2 \pm 12.0$ and $27.9 \pm 16.6 \%$,

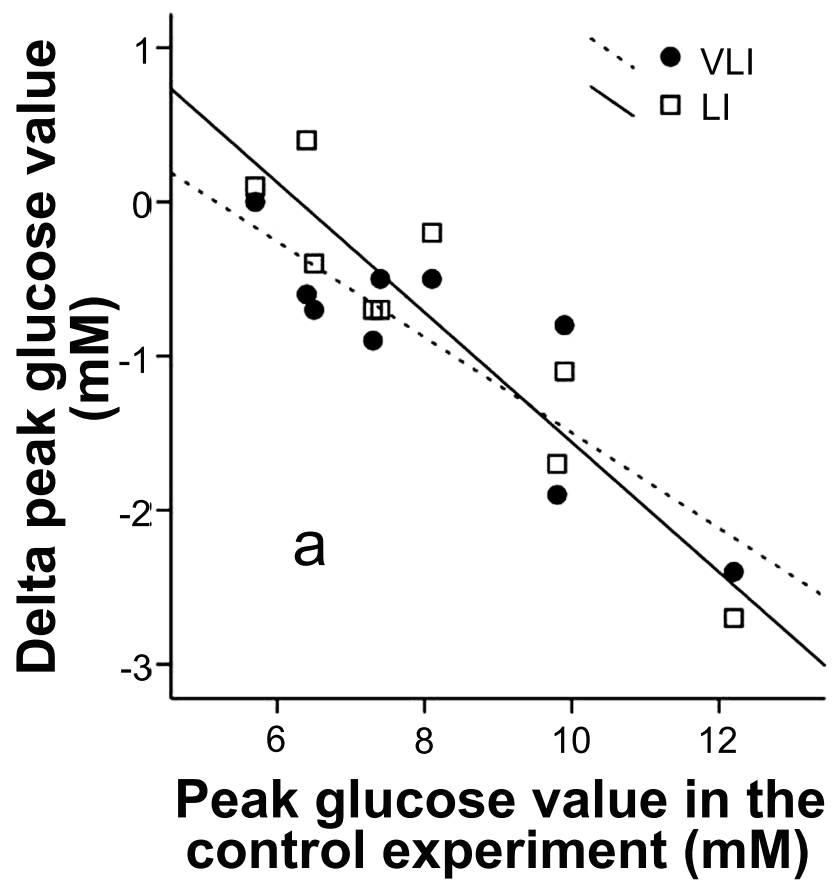

respectively. After Bonferroni correction neither AUC, IAUC nor peak value differed significantly between the LI and control experiment $(\mathrm{p}=0.033, \mathrm{p}=0.030$ and $\mathrm{p}=0.026$, respectively), but still we found decreases of $\sim 20 \%$. Because of missing data the calculations of AUC and IAUC for insulin are based on eight subjects. No significant differences were found between the two exercise experiments in neither glucose nor insulin.

For both glucose and insulin we found strong relationships between the rise observed in the control experiment and the decrease caused by exercise in the VLI and LI experiments. The peak value on the control day (Day I) vs. delta peak values (Day II and III) are shown in Fig. (2a) (glucose) and Fig. (2b) (insulin). Correlation coefficients for peak glucose values were $r=-0.87(p=0.003)$ and $r=-0.93$ $(\mathrm{p}<0.001)$ for the VLI and LI experiment, respectively. Corresponding relationships for peak insulin values were $\mathrm{r}=$ $-0.90(p=0.001)$ and $r=-0.83(p=0.006)$. Strong relationships were also found for concentration after $30 \mathrm{~min}$ of exercise, AUC and IAUC, in which the correlation coefficients for glucose were $r=-0.93(p<0.001), r=-0.91(p=0.001)$ and $r=-0.59(\mathrm{NS})$ respectively in the VLI experiment, and $\mathrm{r}$ $=-0.90(\mathrm{p}=0.001), \mathrm{r}=-0.86(\mathrm{p}=0.003)$ and $\mathrm{r}=-0.54(\mathrm{NS})$ in the LI experiment. Relationships for concentration after $30 \mathrm{~min}$ of exercise, AUC and IAUC for insulin were $\mathrm{r}=$ $0.57(\mathrm{NS}), \mathrm{r}=-0.89(\mathrm{p}=0.003)$ and $\mathrm{r}=-0.92(\mathrm{p}=0.001)$ respectively in the VLI experiment, and $\mathrm{r}=-0.52(\mathrm{NS}), \mathrm{r}=-$ $0.86(\mathrm{p}=0.006)$ and $\mathrm{r}=-0.91(\mathrm{p}=0.003)$ in the LI experiment. Because of missing data the calculations of AUC and IAUC for insulin are based on eight subjects.

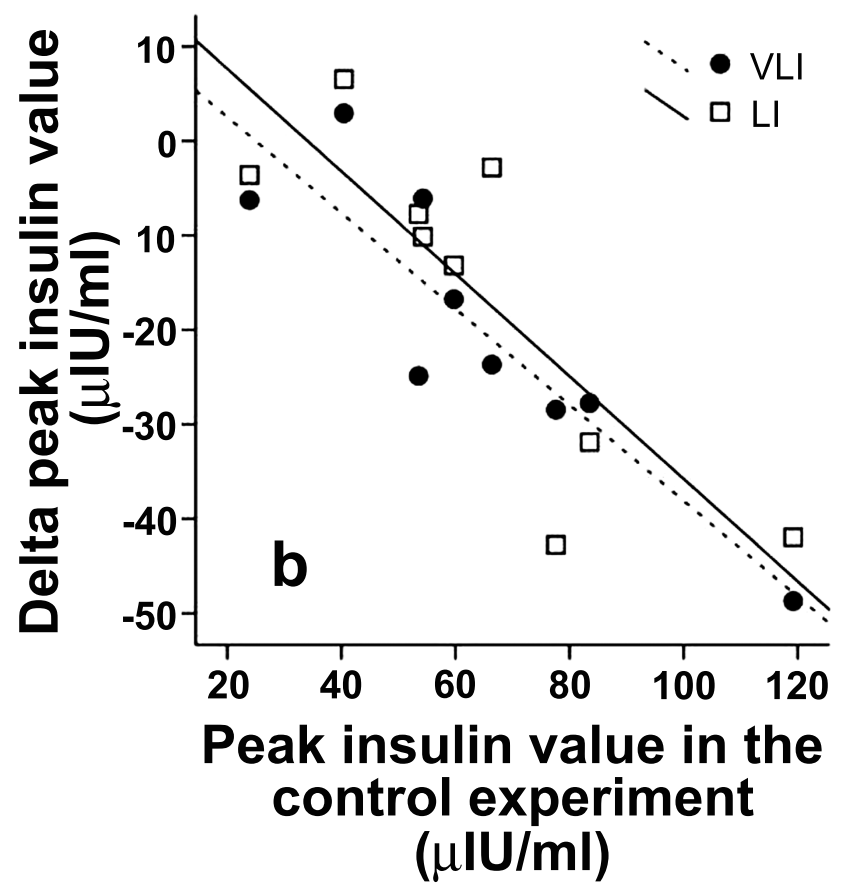

Fig. (2). Relationships between peak value in the control experiment and peak values in the two exercise experiments performed in the study, for glucose (a) and insulin (b). Nine subjects ([mean \pm SD] 37,3 $\pm 12,2$ years, 77,8 $\pm 14,9 \mathrm{~kg}, 174,9 \pm 7,5 \mathrm{~cm}, 5,0 \pm 2,6$ hours of physical activity per week) participated in three diet experiments. Commencing after an overnight fast the same subjects ate cornflakes (1 g carbohydrate per kg body weight) and then rested, or did 30 min very light exercise $\left(58,7 \pm 6,2 \%\right.$ of $\left.\mathrm{HR}_{\max }\right)$ (VLI; •), or 30 min light exercise $(66,6$ $\pm 10,6 \%$ of $\left.\mathrm{HR}_{\max }\right)(\mathrm{LI} ; \square)$ in the time period 15 to $45 \mathrm{~min}$. Strong correlations were found between the peak value the control day (without exercise) and the reduction in the peak value caused by exercise, observed for both glucose (VLI: $r=-0,87(p=0,003)$; LI: $r=-0,93$ ( $p<$ $0,001))$ and insulin (VLI: $r=-0,90(p=0,001)$; LI: $r=-0,83(p=0,006))$. Dots are individual values. The study was carried out at The Norwegian School of Sport Sciences in Oslo, spring 2006. 


\section{DISCUSSION AND CONCLUSION}

The main findings in the present study were that $30 \mathrm{~min}$ of very light and light bicycle exercise ( 59 to $67 \%$ of $\mathrm{HR}_{\max }$ ) performed immediately after a high glycaemic breakfast blunted the rise in blood glucose and insulin, and caused a delayed and attenuated increase. Additionally, the effects of the work performed at the two intensities were strongly associated with each and every individual's level of rise in both glucose and insulin in the absence of post meal exercise.

We found a decrease in blood glucose of $24.5 \pm 21.2$ and $25.8 \pm 19.8 \%$ after $30 \mathrm{~min}$ of exercise for the VLI and LI experiment, respectively, compared to control. This seems to be in accordance with the results of Høstmark et al. [20] who found decreases in the range of $\sim 20$ to $30 \%$ exercising at $\sim 70 \%$ of $\mathrm{HR}_{\max }$, using the same protocol as this study. Unfortunately, Høstmark et al. [20] did not measure insulin levels. However, Nelson et al. [19] reported decreases in insulin in excess of $50 \%$, exercising for $45 \mathrm{~min}$ at $~ 70 \%$ of $\mathrm{HR}_{\max }$, starting $30 \mathrm{~min}$ after breakfast. This is in agreement with our findings of a decrease in insulin after $30 \mathrm{~min}$ of exercise by $53.4 \pm 31.4$ and $68.2 \pm 21.0 \%$ in the VLI and LI experiment respectively, compared to control. Furthermore, the second rise in glucose and insulin found when the exercise terminated are also in agreement with Høstmark et al. [20] and Nelson et al. [19]. Hence, the present findings are in accordance with earlier findings. We have no data to speculate about the mechanisms involved, but it is suggested that decreased glycaemia during exercise is due to increased uptake and utilization of glucose by muscles [25-29].

In an attempt to interpret our results we have to compare our findings with studies using drugs to lower postprandial glycaemia. However, longitudinal studies reporting on health benefits of acarbose have not measured the acute effect of the drugs on glucose and insulin [9-12], which makes the comparison difficult. However, other studies have examined the acute effect of a single administration of acarbose on glycaemia and insulinaemia. The findings are a $\sim 9$ to 31 and $\sim 4$ to $39 \%$ decrease in glucose and insulin respectively, in type 2 diabetics [30-32]. To our knowledge, only one study has reported on the acute effect of acarbose in healthy subjects [33]. Kageyama et al. [33] examined the effect of 50 and $100 \mathrm{mg}$ acarbose in relation to placebo taken three times a single day, in a crossover design in 20 subjects $(26.4 \pm 4.6$ years). The effect was a decrease of $0-12$ and $24-38 \%$ in glucose and insulin concentration, respectively. Corresponding decreases in the present study were $2-10$ and $20-28 \%$. As the effect of exercise in the present study is in the same range as the effect of acarbose in the study by Kageyama et al. [33], we think light exercise in the postprandial period may provide similar health benefits as acarbose. Yet, a problem with physical activity is 'the size of the pill', which many people probably would find hard to swallow. Hence, because of the effort it takes, we do not think most people are willing to be physically active $30 \mathrm{~min}$ after every meal every day for extended periods of time. Based on this, our opinion is that light exercise in the postprandial period probably can provide a health benefit, but should not be recommended as a complete substitute for drugs having similar effect. However, it is important to recognize that exercise in addition to the acute glycaemia-reducing effect, also can provide a wide range of long-lasting health benefits, like qualitative changes in muscles or body composition [34, 35].

We found strong relationships between the rise in glucose and insulin in the control experiment and corresponding lowering effect of post meal exercise, at both intensities. Based on Elahi \& Muller's [21] findings that glucose tolerance decreases along with older age (probably mostly because of inactivity and not age per se), it would appear that exercise in the postprandial period has a greater blood glucose reducing effect in middle-aged and elderly people than at younger age, as was previously shown by Høstmark et al. [20]. One interpretation of this observation is that people at most risk of morbidity and mortality of diabetes and cardiovascular diseases, are probably also those who would benefit the most being physically active after a meal.

At no point of time we found differences between the two exercise experiments. However, peak value for glucose, together with AUC, IAUC and peak value for insulin were significantly lowered only in the VLI experiment, compared to control. Yet, it should be noticed that differences in both the VLI and LI experiments are of about the same magnitude, but differences between the LI and control experiment just missed being significant after Bonferroni correction $(\mathrm{p}=$ 0.026 to 0.040 ). Hence, we consider differences between the two exercise intensities to be minimal, but notice with some surprise that if one of the intensities should be the better, it is the lowest one $\left(58.7 \pm 6.2 \%\right.$ of $\mathrm{HR}_{\max }$, rate of perceived exertion of $10.1 \pm 0.9)$. However, this can be explained by a higher catecholamine secretion during heavier work [36], which in turn would stimulate a higher glycolysis by the liver and increased blood glycaemia.

Overall, we consider our findings to be in accordance with findings of Achten \& Jeukendrup [23], who did not find any difference in postprandial glycaemia or insulinaemia as a result of exercise intensities between $\sim 55$ and $90 \%$ of maximal oxygen consumption. This means that exercise of an intensity that is not considered high enough to increase aerobic capacity [37], can have important glycaemialowering effects. This finding is interesting and positive of two reasons. Firstly, lower intensity exercise cause less strain to be put upon the body and hence, cause less musculoskeletal injuries and gives a higher number of susceptible individuals the opportunity to take part in physical activity. Secondly, light exercise are easier to keep up trough extended periods of time, compared to heavier exercise [37, 38]. For people who find bicycle-exercise difficult, it should be noticed that walking at self-paced speed is generally carried out at intensities between $\sim 60$ and $70 \%$ of $\mathrm{HR}_{\max }$ (Borg rating of $\sim 10$ to 11) [39-44], which corresponds very well to the intensity found to be effective in lowering postprandial glycaemia and insulinaemia in the present study. Even though walking was not examined in the present study, we consider it very likely that walking should provide the same benefit as do light bicycle exercise.

As we found an effect of even very light intensity exercise $\left(58.7 \pm 6.2 \%\right.$ of $\mathrm{HR}_{\max }$; rate of perceived exertion of $10.1 \pm 0.9$ ), the threshold of effect should be somewhat lower. Hence, further research should determine the lowest intensity which could lower the postprandial rise in glucose and insulin, in addition to study the influence of other vari- 
ables, eg duration, point of start after meal and mode of exercise, type of meal and time of the day.

In conclusion, this study shows that $30 \mathrm{~min}$ of very light and light bicycle exercise ( $\sim 59$ to $67 \%$ of $\mathrm{HR}_{\max }$ ) performed immediately after a high glycaemic breakfast can blunt the rise in blood glucose and insulin. The effect of post meal light work is strongly associated with each and every individual's level of rise in both glucose and insulin. Hence, individuals with impaired glucose tolerance, who are at most risk of developing type 2 diabetes and early death, are also those who probably would benefit the most of after-meal physically activity. Interestingly, there is an old Chinese saying: "One hundred steps after a meal make you live to be 99 years old".

\section{ACKNOWLEDGMENT}

We gratefully acknowledge the technical assistance of Eva Kristensen.

\section{REFERENCES}

[1] Dunstan DW, Zimmet PZ, Welborn TA, et al. The rising prevalence of diabetes and impaired glucose tolerance -the Australian diabetes, obesity and lifestyle study. Diabetes Care 2002; 25(5): 829-34.

[2] Wild S, Roglic G, Green A, Sicree R, King H. Global prevalence of diabetes -estimates for the year 2000 and projections for 2030 . Diabetes Care 2004; 27(5): 1047-53.

[3] Brand-Miller JC. Postprandial glycemia, glycemic index, and the prevention of type 2 diabetes. Am J Clin Nutr 2004; 80: 243-4.

[4] DECODE Study Group. Is the current definition for diabetes relevant to mortality risk from all causes and cardiovascular and noncardiovascular diseases? Diabetes Care 2003; 26(3): 688-96.

[5] Levitan EB, Song Y, Ford ES, Liu S. Is nondiabetic hyperglycemia a risk factor for cardiovaskular disease? Arch Intern Med 2004; 164: 2147-55.

[6] Shaw JE, Hodge AM, de Courten M, Chitson P, Zimmet PZ. Isolated post-challenge hyperglycaemia confirmed as a risk factor for mortality. Diabetologia 1999; 42: 1050-4.

[7] Temelkova-Kurktschiev TS, Koehler C, Henkel E, Leonhardt W, Fuecker K, Hanefeld M. Postchallenge plasma glucose and glycemic spikes are more strongly associated with atherosclerosis than fasting glucose or $\mathrm{HbA}_{1 \mathrm{C}}$ level. Diabetes Care 2000; 23(12): 18304.

[8] Cavalot F, Petrelli A, Traversa M, et al. Postprandial blood glucose is a stronger predictor of cardiovascular events than fasting blood glucose in type 2 diabetes mellitus, particularly in women: lessons from the San Luigi Gonzaga diabetes study. J Clin Endocrinol Metab 2006; 91: 813-9.

[9] Chiasson J, Josse RG, Gomis R, Hanefeld M, Karasik A, Laakso M. Acarbose for prevention of type 2 diabetes mellitus: the STOPNIDDM randomised trail. Lancet 2002; 359: 2072-7.

[10] Chiasson J, Josse RG, Gomis R, Hanefeld M, Karasik A, Laakso M. Acarbose treatment and the risk of cardiovascular disease and hypertension in patients with impaired glucose tolerance -the STOP-NIDDM trail. JAMA 2003; 290(4): 486-94.

[11] Hanefeld M, Chiasson J, Koehler C, Henkel E, Schaper F, Tamelkova-Kurktschiev T. Acarbose slows progression of intima-media thickness of the carotid arteries in subjects with impaired glucose tolerance. Stroke 2004; 35: 1073-8.

[12] Hanefeld M, Cagatay M, Petrowitsch T, Neuser D, Petzinna D, Rupp M. Acarbose reduces the risk for myocardial infarction in type 2 diabetic patients: meta-analysis of seven long-term studies. Eur Heart J 2004; 25: 10-6.

[13] Delorme S, Chiasson J. Acarbose in the prevention of cardiovascular disease in subjects with impaired glucose tolerance and type 2 diabetes mellitus. Curr Opin Pharmacol 2005; 5: 184-9.

[14] Ceriello A. Postprandial hyperglycemia and diabetes complications -is it time to treat? Diabetes 2005; 54: 1-7.

[15] Leiter LA, Ceriello A, Davidson JA, et al. Postprandial glucose regulation: new data and new implications. Clin Ther 2005; 27(Suppl B): S42-56.
[16] Zeymer U. Cardiovascular benefits of acarbose in impaired glucose tolerance and type 2 diabetes. Int J Cardiol 2006; 107: 11-20.

[17] Charpentier G, Riveline J, Dardari D, Varroud-Vial M. Should postprandial hyperglycaemia in prediabetic and type 2 diabetic patients be treated? Drugs 2006; 66(3): 273-86.

[18] Rendell MS, Jovanovic L. Targeting postprandial hyperglycemia. Metabolism 2006; 55: 1263-81.

[19] Nelson JD, Poussier P, Marliss EB, Albisser AM, Zinman B. Metabolic response of normal man and insulin-infused diabetics to postprandial exercise. Am J Physiol 1982; 242(5): 309-16.

[20] Høstmark AT, Ekeland GS, Beckstrøm AC, Meen HD. Postprandial light physical activity blunts the blood glucose increase. Prev Med 2006; 42: 369-71.

[21] Elahi D, Muller DC. Carbohydrate metabolism in the elderly. Eur J Clin Nutr 2000; 54(Suppl 3): S112-S20.

[22] Ruzzin J, Jensen J. Contraction activates glucose uptake and glycogen synthase normally in muscles from dexamethasone-treated rats. Am J Physiol Endocrinol Metab 2005; 289: E241-50.

[23] Achten J, Jeukendrup AE. Effects of pre-exercise ingestion of carbohydrate on glycaemic and insulinaemic responses during subsequent exercise at differing intensities. Eur J Appl Physiol 2003; 88: 466-71.

[24] Foster-Powell K, Holt SHA, Brand-Miller JC. International table of glycemic index and glycemic load values: 2002. Am J Clin Nutr 2002; 75: 5-56.

[25] Ahlborg G, Felig P. Influence of glucose ingestion on fuelhormone response during prolonged exercise. J Appl Physiol 1976; 41(5): 683-8.

[26] Ahlborg G, Felig P. Substrate utilization during prolonged exercise preceded by ingestion of glucose. Am J Physiol 1977; 233(3): E188-94.

[27] Kirwan JP, O’Gorman DJ, Cyr-Campbell D, Campbell WW, Yarasheski KE, Evans WJ. Effects of a moderate glycemic meal on exercise duration and substrate utilization. Med Sci Sports Exerc 2001; 33(9): 1517-23.

[28] Marmy-Conus N, Fabris S, Proietto J, Hargreaves M. Preexercise glucose ingestion and glucose kinetics during exercise. J Appl Physiol 1996; 81(2): 853-7.

[29] Wu CL, Nicholas C, Williams C, Took A, Hardy L. The influence of high carbohydrate meals with different glycaemic indices on substrate utilisation during subsequent exercise. Br J Nutr 2003; 90(6): 1049-56.

[30] Shimabukuro M, Higa N, Chinen I, Yamakawa K, Takasu N. Effects of a single administration of Acarbose on postprandial glucose excursion and endothelial dysfunction in type 2 diabetic patients: A randomized crossover study. J Clin Endocrinol Metab 2006; 91(3): 837-42.

[31] Mooradian AD, Albert SG, Wittry S, Chehade J, Kim J, Bellrichard B. Dose-response profile of Acarbose in older subjects with type 2 diabetes. Am J Med Sci 2000; 319(5): 334-7.

[32] Rosak C, Nitzsche G, Konig P, Hofmann U. The effect of the timing and the administration of Acarbose on postprandial hyperglycaemia. Diabet Med 1995; 12: 979-84.

[33] Kageyama S, Nakamichi N, Sekino H, Nakano S. Comparison of the effects of Acarbose and Voglibose in healthy subjects. Clin Ther 1997; 19(4): 720-9.

[34] Ivy JL, Zderic TW, Fogt DL. Prevention and treatment of NonInsulin-Dependent Diabetes Mellitus. In: Holloszy JO Ed. Exerc Sport Sci Rev. New York: Lippincott Williams \& Wilkins 1999; 135 .

[35] Dela F, Vaag AA. Fysisk traening til behandlinga af type 2diabetes. Ugeskr Laeger 2000; 162(15): 2185-9.

[36] Marliss EB, Vranic M. Intense exercise has unique effects on both insulin release and its roles in glucoregulation -implications for diabetes. Diabetes 2002; 51(Suppl 1): 271-83.

[37] American College of Sports Medicine (ACSM's). Guidelines for exercise testing and prescription. 6 ed. USA: Lippincott Williams \& Wilkins; 2000.

[38] American College of Sports Medicine (ACSM's). The recommended quantity and quality of exercise for developing and maintaining cardiorespiratory and muscular fitness, and flexibility in healthy adults. Med Sci Sports Exerc 1998; 30(6): 975-91.

[39] Murtagh EM, Boreham CAG, Murphy MH. Speed and exercise intensity of recreational walkers. Prev Med 2002; 35: 397-400. 
[40] Spelman CC, Pate RR, Macera CA, Ward DS. Self-selected exercise intensity of habitual walkers. Med Sci Sports Exerc 1993; 25(10): 1174-9.

[41] Withers RT, Brooks AG, Gunn SM, Plummer JL, Gore CJ, Cormack J. Self-selected exercise intensity during household/garden activities and walking in 55 to 65 -year-old females. Eur J Appl Physiol 2006; 97: 494-504.

[42] Brooks AG, Gunn SM, Withers RT, Gore CJ, Plummer JL. Predicting walking METs and energy expenditure from speed or accelerometry. Med Sci Sports Exerc 2005; 37(7): 1216-23.
[43] Browning RC, Kram R. Energetic cost and preferred speed of walking in obese vs. normal weight women. Obes Res 2005; 13: 891-9.

[44] Pintar JA, Robertson RJ, Kriska AM, Nagle E, Goss FL. The influence of fitness and body weight on preferred exercise intensity. Med Sci Sports Exerc 2006; 38(5): 981-8.

() Aadland and Høstmark; Licensee Bentham Open.

This is an open access article licensed under the terms of the Creative Commons Attribution Non-Commercial License (http://creativecommons.org/licenses/by-nc/3.0/) which permits unrestricted, non-commercial use, distribution and reproduction in any medium, provided the work is properly cited. 